\title{
On the strong convergence of multiple ordinary integrals to multiple Stratonovich integrals
}

\author{
Xavier Bardina ${ }^{\dagger}$ and Carles Rovira*
}

${ }^{\dagger}$ Departament de Matemàtiques, Facultat de Ciències, Edifici C, Universitat Autònoma de Barcelona, 08193 Bellaterra. Xavier.Bardina@uab.cat

*Departament de Matemàtiques i Informàtica, Universitat de Barcelona, Gran Via 585, 08007 Barcelona. carles.rovira@ub.edu

\begin{abstract}
Given $\left\{W^{(m)}(t), t \in[0, T]\right\}_{m \geq 1}$ a sequence of approximations to a standard Brownian motion $W$ in $[0, T]$ such that $W^{(m)}(t)$ converges almost surely to $W(t)$ we show that, under regular conditions on the approximations, the multiple ordinary integrals with respect to $d W^{(m)}$ converge to the multiple Stratonovich integral. We are integrating functions of the type$$
f\left(x_{1}, \ldots, x_{n}\right)=f_{1}\left(x_{1}\right) \ldots f_{n}\left(x_{n}\right) I_{\left\{x_{1} \leq \ldots \leq x_{n}\right\}},
$$

where for each $i \in\{1, \ldots, n\}, f_{i}$ has continuous derivatives in $[0, T]$. We apply this result to approximations obtained from uniform transport processes.
\end{abstract}

\section{Introduction}

Let $W:=\{W(t), t \geq 0\}$ be a standard Brownian motion and let $\left\{W^{(m)}(t), t \in\right.$ $[0, T]\}_{m \geq 1}$ a sequence of approximations to $W$ in $[0, T]$ such that $W^{(m)}(t)$ converge almost surely to $W(t)$ as $m$ tends to $\infty$. It is well known that if the approximations are of bounded variation and continuous then, almost surely

$$
\begin{gathered}
\lim _{m \rightarrow \infty} \int_{0}^{t} W^{(m)}(s) d W^{(m)}(s)=\lim _{m \rightarrow \infty} \frac{\left(W^{(m)}(t)\right)^{2}}{2}=\frac{(W(t))^{2}}{2} \\
=\int_{0}^{t} W(s) d W(s)+\frac{t}{2}=\int_{0}^{t} W(s) d^{\circ} W(s)
\end{gathered}
$$

$t \in[0, T]$, where $d W$ denotes the Itô integral and $d^{\circ} W$ is the Stratonovich one.

Wong and Zakai [13] extended this result obtaining relations between limits of integrals of the type $\int_{0}^{t} \psi\left(W^{(m)}(s), s\right) d W^{(m)}(s)$ and $\int_{0}^{t} \psi(W(s), s) d^{\circ} W(s)$.

${ }^{*}$ The authors are supported by the grant PGC2018-097848-B-I00. 
More precisely, assuming that the approximations are of bounded variation, continuous and for each $m$ can be bounded by a finite random variable and that $\psi(\eta, s)$ has continuous partial derivatives with respect to $\eta$ and $s$, they proved that, for $t \in[0, T]$, almost surely,

$$
\begin{aligned}
\lim _{m \rightarrow \infty} \int_{0}^{t} & \psi\left(W^{(m)}(s), s\right) d W^{(m)}(s) \\
& =\int_{0}^{t} \psi(W(s), s) d W(s)+\frac{1}{2} \int_{0}^{t} \frac{\partial \psi}{\partial \eta}(W(s), s) d s \\
& =\int_{0}^{t} \psi(W(s), s) d^{\circ} W(s) .
\end{aligned}
$$

Gorostiza and Griego [10] applied this result to get strong approximations to stochastic integrals using uniform transport processes as approximations to Brownian motion. They also obtain the corresponding rate of convergence.

Our aim is to extend this result to study the problem of the strong convergence, as $m$ tends to $\infty$, of the multiple integral processes

$$
\left\{\int_{0}^{t} \int_{0}^{s_{n}} \ldots \int_{0}^{s_{2}} f_{n}\left(s_{n}\right) \ldots f_{1}\left(s_{1}\right) d W^{(m)}\left(s_{1}\right) \ldots d W^{(m)}\left(s_{n}\right), t \in[0, T]\right\},
$$

where for each $i \in\{1, \ldots, n\}, f_{i}$ has continuous derivatives in $[0, T]$. We will obtain the almost sure convergence to the multiple Stratonovich integral

$$
\left\{\int_{0}^{t} \int_{0}^{s_{n}} \ldots \int_{0}^{s_{2}} f_{n}\left(s_{n}\right) \ldots f_{1}\left(s_{1}\right) d^{\circ} W\left(s_{1}\right) \ldots d^{\circ} W\left(s_{n}\right), t \in[0, T]\right\} .
$$

We will also apply this convergence to approximations obtained from uniform transport processes. This kind of approximations of multiple integrals has been studied by Bardina and Jolis 3] where they obtain a weak convergence result. Multiple stochastic integrals are useful to represent functionals of Wienner proces and they appear in stochastic Taylor expansions.

The processes usually called uniform transport processes can be presented as

$$
W^{(m)}(t)=m^{\frac{1}{2}}(-1)^{A} \int_{0}^{t}(-1)^{N(u m)} d u,
$$

where $\{N(t), t \geq 0\}$ is a standard Poisson process and $A \sim \operatorname{Bernoulli}\left(\frac{1}{2}\right)$ independent of the Poisson process $N$.

There is some literature related with strong convergence using approximations based on realizations of these processes. Griego, Heath and Ruiz-Moncayo [1] showed that these processes converge strongly and uniformly on bounded time intervals to Brownian motion. In [9] Gorostiza and Griego extended the result to diffusions. Again Gorostiza and Griego [10] and Csörgö and Horváth 4 obtained a rate of convergence. Garzón, Gorostiza and León [6] defined a sequence of processes that converges strongly to fractional Brownian motion uniformly on bounded intervals, for any Hurst parameter $H \in(0,1)$. In [7] and 
[8] the same authors deal with subfractional Brownian motion and fractional stochastic differential equations. Garzón, Torres and Tudor [5] also studied the Rosenblatt process. In [2] we get strong approximations to Brownian sheet and in [1] for complex Brownian motion.

The proof of our results follows the ideas in 11, using Itô's formula as a main tool. It is important to get an expression of the multiple Stratonovich integral and the relation with the multiple Itô integral well-posed for our problem. We give a detailed expression of this relationship, inspired by the well-known $\mathrm{Hu}-$ Meyer formula and the work of Kloeden and Platen [12].

The paper is organized in the following way. Section 2 is devoted to define the processes and to give the main results. In Section 3 we study the relationship between Itô integral and Stratonovich integral. In Section 4 we give the proof of the main theorem.

\section{Notations and main result}

Consider

$$
f\left(x_{1}, \ldots, x_{n}\right)=f_{1}\left(x_{1}\right) \ldots f_{n}\left(x_{n}\right) I_{\left\{x_{1} \leq \ldots \leq x_{n}\right\}},
$$

where $f_{i} \in L^{2}[0, T]$ for all $i \in\{1, \ldots, n\}$. From Lemma A.1 and A.2 of [3] it is known that $f$ is Stratonovich integrable and the process

$$
\begin{aligned}
I_{n}^{S}(t) & :=I_{n}^{S}\left(f_{1}, \ldots, f_{n}\right)(t) \\
& =\int_{0}^{t} \int_{0}^{s_{n}} \ldots \int_{0}^{s_{2}} f_{n}\left(s_{n}\right) \ldots f_{1}\left(s_{1}\right) d^{\circ} W\left(s_{1}\right) \ldots d^{\circ} W\left(s_{n}\right)
\end{aligned}
$$

has a version with continuous paths. Moreover, there exist the following iterated simple integrals

$$
Y_{k}(t)=\int_{0}^{t} f_{k}(s) Y_{k-1}(s) d^{\circ} W(s),
$$

for $k \in\{2, \ldots, n\}$, where $Y_{1}(t)=\int_{0}^{t} f_{1}(s) d^{\circ} W(s)$ and all these integrals have a continuous version and $Y_{n}$ coincides with $I_{n}^{S}$.

Set, for each $m, W^{(m)}(t)$ a sequence of approximations to $W(t)$ such that $W^{(m)}(t)$ is of bounded variation, continuous and converges a.s. to $W(t)$ as $m \rightarrow \infty$. Then, we define the ordinary multiple integral

$$
\begin{aligned}
J_{n}^{(m)}(t) & :=J_{n}^{(m)}\left(f_{1}, \ldots, f_{n}\right)(t) \\
& =\int_{0}^{t} \int_{0}^{s_{n}} \ldots \int_{0}^{s_{2}} f_{n}\left(s_{n}\right) \ldots f_{1}\left(s_{1}\right) d W^{(m)}\left(s_{1}\right) \ldots d W^{(m)}\left(s_{n}\right) .
\end{aligned}
$$

Obviously, we can consider the iterated integrals

$$
J_{k}^{(m)}\left(f_{1}, \ldots, f_{k}\right)(t)=\int_{0}^{t} f_{k}(s) J_{k-1}^{(m)}\left(f_{1}, \ldots, f_{k-1}\right)(s) d W^{(m)}(s),
$$


for $k \in\{2, \ldots, n\}$ and where

$$
J_{1}^{(m)}\left(f_{1}\right)(t)=\int_{0}^{t} f_{1}(s) d W^{(m)}(s) .
$$

Following the ideas of Wong and Zakai [13, we state the set of hypothesis $(\mathrm{H})$ on $W^{(m)}$ as follows:

(H1) almost surely, $W^{(m)}$ are continuous and of bounded variation,

(H2) almost surely, $\lim _{m \rightarrow \infty} W^{(m)}(s)=W(s)$ for all $s \in[0, T]$,

(H3) for almost all $\omega$, there exists $m_{0}(\omega)$ and $K(\omega)$, both finite, such that for all $m>m_{0}$ and all $s \in[0, T], W^{(m)}(s, \omega) \leq K(\omega)$,

(H4) $\lim _{m \rightarrow \infty} \sup _{s \in[0, T]}\left|W^{(m)}(s)-W(s)\right|=0$ almost surely,

Then, the main theorem reads as follows:

Theorem 2.1. Assume that $f:[0, T]^{n} \rightarrow \mathbb{R}$ is a function

$$
f\left(x_{1}, \ldots, x_{n}\right)=f_{1}\left(x_{1}\right) \ldots f_{n}\left(x_{n}\right) I_{\left\{x_{1} \leq \ldots \leq x_{n}\right\}},
$$

such that for each $i \in\{1, \ldots, n\}, f_{i}$ has continuous derivatives in $[0, T]$. Let $\left(W^{(m)}\right)$ a family of approximations of $W$ that satisfy hypothesis (H1), (H2) and (H3). Then, almost surely,

$$
\lim _{m \rightarrow \infty} J_{n}^{(m)}(t)=I_{n}^{S}(t)
$$

for all $t \in[0, T]$.

If $\left(\mathrm{H}_{4}\right)$ is also true, then

$$
\lim _{m \rightarrow \infty} \sup _{s \in[0, T]}\left|J_{n}^{(m)}(s)-I_{n}^{S}(s)\right|=0
$$

almost surely,

We can give now our theorem using approximations based on uniform transport process.

Theorem 2.2. Consider $W^{(m)}:=\left\{W^{(m)}(t), t \in[0, T]\right\}$ versions of the uniform transport process (1) on the same probability space as a Brownian motion $\{W(t), t \in[0, T]\}$ such that

$$
\lim _{m \rightarrow \infty} \sup _{s \in[0, T]}\left|W^{(m)}(s)-W(s)\right|=0
$$

almost surely. Assume that $f:[0, T]^{n} \rightarrow \mathbb{R}$ is a function

$$
f\left(x_{1}, \ldots, x_{n}\right)=f_{1}\left(x_{1}\right) \ldots f_{n}\left(x_{n}\right) I_{\left\{x_{1} \leq \ldots \leq x_{n}\right\}},
$$

such that for each $i \in\{1, \ldots, n\}, f_{i}$ has continuous derivatives in $[0, T]$. Then

$$
\lim _{m \rightarrow \infty} \sup _{s \in[0, T]}\left|J_{n}^{(m)}(s)-I_{n}^{S}(s)\right|=0
$$

almost surely, 
Remmark 2.3. Our result can be used to simulate multiple Stratonovich stochastic integrals $I_{n}^{S}$ using as an approximation $J_{n}^{(m)}(t)$. In order to compute $J_{n}^{(m)}(t)$ we can use the following decomposition that we proof in Lemma 4.1:

$$
\begin{aligned}
& J_{n}^{(m)}(t)=\sum_{k=1}^{n}(-1)^{k+1} J_{n-k}^{(m)}(t) \frac{\left(W^{(m)}(t)\right)^{k}}{k !}\left(\prod_{l=1}^{k} f_{n+1-l}\right)(t) \\
& +\sum_{k=1}^{n}(-1)^{k} \int_{0}^{t} J_{n-k}^{(m)}(s) \frac{\left(W^{(m)}(s)\right)^{k}}{k !}\left(\prod_{l=1}^{k} f_{n+1-l}\right)^{\prime}(s) d s,
\end{aligned}
$$

for any $t \in[0, T]$ and with $J_{0}^{(m)}(t)=1$.

Remmark 2.4. In Bardina and Jolis [3] the authors prove the weak convergence, when $m$ tends to infinty, of the multiple integral processes $\left\{J_{n}^{(m)}(t), t \in\right.$ $[0, T]\}$ in the space of the real continuous functions when $f_{i} \in L^{2}[0, T]$ for all $i \in\{1, \ldots, n\}$. To obtain the almost sure convergence we need to assume stronger conditions on functions $f_{i}$ in order to be able to use Itô formula. The derivability hypothesis that we assume is similar to the one used in the paper of Wong and Zakai [13].

Bardina and Jolis [3] also study the weak limit for other classes of integrals. Particularly, they consider the limit of integrals

$$
\left\{\int_{0}^{t} \int_{0}^{s_{n}} \ldots \int_{0}^{s_{2}} f\left(s_{1}, \ldots, s_{n}\right) d W^{(m)}\left(s_{1}\right) \ldots d W^{(m)}\left(s_{n}\right), t \in[0, T]\right\},
$$

when $f$ is given by a multimeasure. Notice that in this case the almost sure convergence is obtained easily from Theorem 3.1 in [3] and the almost sure convergence in [11].

\section{Multiple Itô and Stratonovich integrals}

The relation between simple Itô and Stratonovich integrals have been studied deeply. For instance, it is well known that if $X:=\{X(t), t \geq 0\}$ is an adapted continuous process then

$$
\int_{0}^{t} X(s) d^{\circ} W(s)=\int_{0}^{t} X(s) d W(s)+\frac{1}{2}[X, W]_{t} .
$$

where $[$,$] denotes the quadratic covariation. Particularly, if$

$$
X(t)=X(0)+\int_{0}^{t} u(s) d W(s)+\int_{0}^{t} v(s) d s
$$

where $\{u(t), t \geq 0\}$ and $\{v(t), t \geq 0\}$ are adapted processes, and $g$ is a differentiable real function, since we can write that

$$
g(t) X(t)=g(0) X(0)+\int_{0}^{t} g(s) d X(s)+\int_{0}^{t} X(s) g^{\prime}(s) d s
$$


we obtain that

$$
\int_{0}^{t} g(s) X(s) d^{\circ} W(s)=\int_{0}^{t} g(s) X(s) d W(s)+\frac{1}{2} \int_{0}^{t} g(s) u(s) d s .
$$

There exists also literature about the relations for the multiple stochastic integral, begining with the well-known Hu-Meyer formula. Here we obtain some results well adapted to our problem following the notation presented by Kloeden and Platen [12.

Let us introduce some notation in order to deal with multiple integrals. We assume again that $f_{i} \in L^{2}[0, T]$ for all $i \in\{1, \ldots, n\}$. Set

$$
\mathcal{G}_{n}:=\left\{\left(\alpha_{1}, \ldots, \alpha_{m}\right) \text { such that } \alpha_{i} \in\{1,2\} \forall i, \sum_{i=1}^{m} \alpha_{i}=n\right\}
$$

Notice that if $\alpha=\left(\alpha_{1}, \ldots, \alpha_{m}\right) \in \mathcal{G}_{n}$ then $\frac{n}{2} \leq m \leq n$. Then for any $\alpha=$ $\left(\alpha_{1}, \ldots, \alpha_{m}\right) \in \mathcal{G}_{n}$, let us define

$$
I_{\left(\alpha_{1}, \ldots, \alpha_{m}\right)}\left(f_{1}, \ldots, f_{n}\right)(t)=\left\{\begin{array}{c}
\int_{0}^{t} f_{n}(s) I_{\left(\alpha_{1}, \ldots, \alpha_{m-1}\right)}\left(f_{1}, \ldots, f_{n-1}\right)(s) d W(s) \\
\text { if } \alpha_{m}=1, \\
\int_{0}^{t} f_{n}(s) f_{n-1}(s) I_{\left(\alpha_{1}, \ldots, \alpha_{m-1}\right)}\left(f_{1}, \ldots, f_{n-2}\right)(s) d s \\
\text { if } \alpha_{m}=2,
\end{array}\right.
$$

with

$$
\begin{aligned}
I_{(1)}\left(f_{1}\right)(t) & =\int_{0}^{t} f_{1}(s) d W(s) \\
I_{(2)}\left(f_{1}, f_{2}\right)(t) & =\int_{0}^{t} f_{2}(s) f_{1}(s) d s .
\end{aligned}
$$

Clearly, under our assumptions, all these integrals are well-defined. Moreover, for $\alpha=(1, \ldots, 1)$ we have the iterated Itô integral that coincides with the classical multiple Itô integral:

$$
I_{(1, \ldots, 1)}\left(f_{1}, \ldots, f_{n}\right)(t)=\int_{0}^{t} \int_{0}^{s_{n}} \ldots \int_{0}^{s_{2}} f_{n}\left(s_{n}\right) \ldots f_{1}\left(s_{1}\right) d W\left(s_{1}\right) \ldots d W\left(s_{n}\right) .
$$

We will also use the notation

$$
I_{n}(t)=I_{n}\left(f_{1}, \ldots, f_{n}\right)(t)=I_{(1, \ldots, 1)}\left(f_{1}, \ldots, f_{n}\right)(t) .
$$

We finish this section with three propositions that give us three differents ways to express the Stratonovich integral $I_{n}^{S}(t)=I_{n}^{S}\left(f_{1}, \ldots, f_{n}\right)(t)$. In the first one we show an expression using the notation we have introduced above. 
Proposition 3.1. Assume that $f_{i} \in L^{2}[0, T]$ for all $i \in\{1, \ldots, n\}$. Then

$$
I_{n}^{S}\left(f_{1}, \ldots, f_{n}\right)(t)=\sum_{\left(\alpha_{1}, \ldots, \alpha_{m}\right) \in \mathcal{G}_{n}} \frac{1}{2^{n-m}} I_{\left(\alpha_{1}, \ldots, \alpha_{m}\right)}\left(f_{1}, \ldots, f_{n}\right)(t),
$$

for any $t \in[0, T]$.

Proof: We will check the equality by induction on $n$. For $n=1$ it is easy to see that

$$
I_{1}^{S}\left(f_{1}\right)(t)=\int_{0}^{t} f_{1}(s) d^{\circ} W(s)=\int_{0}^{t} f_{1}(s) d W(s)=I_{(1)}\left(f_{1}\right)(t)
$$

and that $\left\{\mathcal{G}_{1}\right\}=\{(1)\}$.

Let us assume that it is true until $n$ and we will check wthat happens for $n+1$. Using (2) and the induction hypothesis we have that

$$
\begin{aligned}
& I_{n+1}^{S}\left(f_{1}, \ldots, f_{n}, f_{n+1}\right)(t)=\int_{0}^{t} f_{n+1}(s) I_{n}^{S}\left(f_{1}, \ldots, f_{n}\right)(s) d^{\circ} W(s) \\
& =\int_{0}^{t} f_{n+1}(s)\left(\sum_{\left(\alpha_{1}, \ldots, \alpha_{m}\right) \in \mathcal{G}_{n}} \frac{1}{2^{n-m}} I_{\left(\alpha_{1}, \ldots, \alpha_{m}\right)}\left(f_{1}, \ldots, f_{n}\right)(s)\right) d^{\circ} W(s) \\
& =\sum_{\left(\alpha_{1}, \ldots, \alpha_{m}\right) \in \mathcal{G}_{n}} \frac{1}{2^{n-m}} \int_{0}^{t} f_{n+1}(s) I_{\left(\alpha_{1}, \ldots, \alpha_{m}\right)}\left(f_{1}, \ldots, f_{n}\right)(s) d^{\circ} W(s) .
\end{aligned}
$$

Notice that if $\alpha_{m}=1$, using (4), we can write

$$
\begin{aligned}
& \int_{0}^{t} f_{n+1}(s) I_{\left(\alpha_{1}, \ldots, \alpha_{m}\right)}\left(f_{1}, \ldots, f_{n}\right)(s) d^{\circ} W(s) \\
& =\int_{0}^{t} f_{n+1}(s) I_{\left(\alpha_{1}, \ldots, \alpha_{m}\right)}\left(f_{1}, \ldots, f_{n}\right)(s) d W(s) \\
& \quad+\frac{1}{2} \int_{0}^{t} f_{n+1}(s) f_{n}(s) I_{\left(\alpha_{1}, \ldots, \alpha_{m-1}\right)}\left(f_{1}, \ldots, f_{n-1}\right)(s) d s \\
& =I_{\left(\alpha_{1}, \ldots, \alpha_{m}, 1\right)}\left(f_{1}, \ldots, f_{n}, f_{n+1}\right)(t) \\
& \quad+\frac{1}{2} I_{\left(\alpha_{1}, \ldots, \alpha_{m-1}, 2\right)}\left(f_{1}, \ldots, f_{n-1}, f_{n}, f_{n+1}\right)(t) .
\end{aligned}
$$

On the other hand, using (4), when $\alpha_{m}=2$ it holds that

$$
\begin{aligned}
& \int_{0}^{t} f_{n+1}(s) I_{\left(\alpha_{1}, \ldots, \alpha_{m}\right)}\left(f_{1}, \ldots, f_{n}\right)(s) d^{\circ} W(s) \\
& \quad=\int_{0}^{t} f_{n+1}(s) I_{\left(\alpha_{1}, \ldots, \alpha_{m}\right)}\left(f_{1}, \ldots, f_{n}\right)(s) d W(s) \\
& \quad=I_{\left(\alpha_{1}, \ldots, \alpha_{m}, 1\right)}\left(f_{1}, \ldots, f_{n}, f_{n+1}\right)(t) .
\end{aligned}
$$


Putting together (5), (6) and (7) we finish the proof since

$$
\begin{aligned}
& I_{n+1}^{S}\left(f_{1}, \ldots, f_{n}, f_{n+1}\right)(t) \\
& \quad=\sum_{\left(\alpha_{1}, \ldots, \alpha_{m}\right) \in \mathcal{G}_{n}} \frac{1}{2^{n-m}} I_{\left(\alpha_{1}, \ldots, \alpha_{m}, 1\right)}\left(f_{1}, \ldots, f_{n}, f_{n+1}\right)(t) \\
& \quad+\sum_{\left(\alpha_{1}, \ldots, \alpha_{m}\right) \in \mathcal{G}_{n}, \alpha_{m}=1} \frac{1}{2^{n-m}} \frac{1}{2} I_{\left(\alpha_{1}, \ldots, \alpha_{m-1}, 2\right)}\left(f_{1}, \ldots, f_{n}, f_{n+1}\right)(t) \\
& \quad=\sum_{\left(\alpha_{1}, \ldots, \alpha_{m}\right) \in \mathcal{G}_{n+1}} \frac{1}{2^{n+1-m}} I_{\left(\alpha_{1}, \ldots, \alpha_{m}\right)}\left(f_{1}, \ldots, f_{n}, f_{n+1}\right)(t)
\end{aligned}
$$

Notice that in the last equality we have used that

$$
\begin{aligned}
\{( & \left.\left.\alpha_{1}, \ldots, \alpha_{m}, 1\right) ;\left(\alpha_{1}, \ldots, \alpha_{m}\right) \in \mathcal{G}_{n}\right\} \\
& \cup\left\{\left(\alpha_{1}, \ldots, \alpha_{m-1}, 2\right) ;\left(\alpha_{1}, \ldots, \alpha_{m}\right) \in \mathcal{G}_{n} \text { with } \alpha_{m}=1\right\} \\
= & \left\{\left(\alpha_{1}, \ldots, \alpha_{m+1}\right) \text { with } \alpha_{i} \in\{1,2\} \forall i, \alpha_{m+1}=1, \sum_{i=1}^{m+1} \alpha_{i}=n+1\right\} \\
& \cup\left\{\left(\alpha_{1}, \ldots, \alpha_{m}\right) \text { with } \alpha_{i} \in\{1,2\} \forall i, \alpha_{m}=2, \sum_{i=1}^{m} \alpha_{i}=n+1\right\} \\
= & \mathcal{G}_{n+1} .
\end{aligned}
$$

Now, applying Proposition 3.1 we get an iterative definition of $I_{n}^{S}$ in terms of $I_{n-1}^{S}$ and $I_{n-2}^{S}$.

Proposition 3.2. Assume that $f_{i} \in L^{2}[0, T]$ for all $i \in\{1, \ldots, n\}$. Then

$$
I_{n}^{S}(t)=\int_{0}^{t} f_{n}(s) I_{n-1}^{S}(s) d W_{s}+\frac{1}{2} \int_{0}^{t} f_{n}(s) f_{n-1}(s) I_{n-2}^{S}(s) d s,
$$

for any $t \in[0, T]$. 
Proof: It follows easily from the fact that

$$
\begin{aligned}
& I_{n}^{S}\left(f_{1}, \ldots, f_{n}\right)(t)=\sum_{\left(\alpha_{1}, \ldots, \alpha_{m}\right) \in \mathcal{G}_{n}} \frac{1}{2^{n-m}} I_{\left(\alpha_{1}, \ldots, \alpha_{m}\right)}\left(f_{1}, \ldots, f_{n}\right)(t) \\
& =\sum_{\left(\alpha_{1}, \ldots, \alpha_{m}\right) \in \mathcal{G}_{n}, \alpha_{m}=1} \frac{1}{2^{n-m}} \int_{0}^{t} f_{n}(s) I_{\left(\alpha_{1}, \ldots, \alpha_{m-1}\right)}\left(f_{1}, \ldots, f_{n-1}\right)(s) d W(s) \\
& +\sum_{\left(\alpha_{1}, \ldots, \alpha_{m}\right) \in \mathcal{G}_{n}, \alpha_{m}=2} \frac{1}{2^{n-m}} \\
& \quad \times \int_{0}^{t} f_{n}(s) f_{(n-1)}(s) I_{\left(\alpha_{1}, \ldots, \alpha_{m-1}\right)}\left(f_{1}, \ldots, f_{n-2}\right)(s) d s \\
& =\int_{0}^{t} f_{n}(s) \\
& \quad \times\left(\sum_{\left(\alpha_{1}, \ldots, \alpha_{m-1}\right) \in \mathcal{G}_{n-1}} \frac{1}{2^{n-1-(m-1)}} I_{\left(\alpha_{1}, \ldots, \alpha_{m-1}\right)}\left(f_{1}, \ldots, f_{n-1}\right)(s)\right) d W(s) \\
& +\frac{1}{2} \int_{0}^{t} f_{n}(s) f_{(n-1)}(s) \\
& \quad \times\left(\sum_{\left(\alpha_{1}, \ldots, \alpha_{m-1}\right) \in \mathcal{G}_{n-2},} \frac{1}{2^{n-2-(m-1)}} I_{\left(\alpha_{1}, \ldots, \alpha_{m-1}\right)}\left(f_{1}, \ldots, f_{n-2}\right)(s)\right) d s .
\end{aligned}
$$

In the next Proposition we express $I_{n}^{S}$ using $I_{k}^{S}$ for all $k \in\{1, \ldots, n-1\}$. This Proposition is inspired by the work of Wong and Zakai [13] and it is the key of the proof of our main theorem.

Proposition 3.3. Assume for each $i \in\{1, \ldots, n\}, f_{i}$ has continuous derivatives in $[0, T]$. Then

$$
\begin{aligned}
I_{n}^{S}(t) & =\sum_{k=1}^{n}(-1)^{k+1} I_{n-k}^{S}(t) \frac{W(t)^{k}}{k !}\left(\prod_{l=1}^{k} f_{n+1-l}\right)(t) \\
& -\sum_{k=1}^{n}(-1)^{k} \int_{0}^{t} I_{n-k}^{S}(s) \frac{W(s)^{k}}{k !}\left(\prod_{l=1}^{k} f_{n+1-l}\right)^{\prime}(s) d s,
\end{aligned}
$$

for any $t \in[0, T]$ and where $I_{0}^{S}=1$.

Proof: Let us consider the function $F(x, y, u)=x y f_{n}(u)$. By Itô's formula we can write

$$
\begin{aligned}
& F\left(I_{n-1}^{S}(t), W(t), t\right)=\int_{0}^{t} W(s) f_{n}(s) d I_{n-1}^{S}(s) \\
& \quad+\int_{0}^{t} I_{n-1}^{S}(s) f_{n}(s) d W(s)+\int_{0}^{t} I_{n-1}^{S}(s) W(s) f_{n}^{\prime}(s) d s \\
& \quad+\frac{1}{2} \int_{0}^{t} f_{n}(s) d\left[I_{n-1}^{S}(s), W(s)\right] .
\end{aligned}
$$


Let us check first that

$$
\int_{0}^{t} I_{n-1}^{S}(s) f_{n}(s) d W(s)+\frac{1}{2} \int_{0}^{t} f_{n}(s) d\left[I_{n-1}^{S}(s), W(s)\right]=I_{n}^{S}(t),
$$

Using Proposition 3.2 it suffices to check that

$$
\int_{0}^{t} f_{n}(s) d\left[I_{n-1}^{S}(s), W(s)\right]=\int_{0}^{t} f_{n}(s) f_{n-1}(s) I_{n-2}^{S}(s) d s,
$$

but it is an obvious consequence again of Proposition 3.2. So it is clear that (9) holds.

Then, from (8) and (9) and the definition of $F$ it follows that

$$
\begin{aligned}
I_{n}^{S}(t) & =I_{n-1}^{S}(t) W(t) f_{n}(t)-\int_{0}^{t} W(s) f_{n}(s) d I_{n-1}^{S}(s) \\
& -\int_{0}^{t} I_{n-1}^{S}(s) W(s) f_{n}^{\prime}(s) d s .
\end{aligned}
$$

Let us study now the term

$$
\int_{0}^{t} W(s) f_{n}(s) d I_{n-1}^{S}(s) .
$$

Actually, we will study the more general term

$$
H_{k}^{S}(t):=\int_{0}^{t} \frac{(W(s))^{k}}{k !}\left(\prod_{l=1}^{k} f_{n+1-l}\right)(s) d I_{n-k}^{S}(s) .
$$

From Proposition 3.2 it follows that

$$
\begin{aligned}
H_{k}^{S}(t)= & \int_{0}^{t} \frac{(W(s))^{k}}{k !}\left(\prod_{l=1}^{k} f_{n+1-l}\right)(s) d I_{n-k}^{S}(s) \\
= & \int_{0}^{t} \frac{(W(s))^{k}}{k !}\left(\prod_{l=1}^{k+1} f_{n+1-l}\right)(s) I_{n-k-1}^{S}(s) d W(s) \\
& +\frac{1}{2} \int_{0}^{t} \frac{(W(s))^{k}}{k !}\left(\prod_{l=1}^{k+2} f_{n+1-l}\right)(s) I_{n-k-2}^{S}(s) d s .
\end{aligned}
$$

Consider now the function $F(x, y, u)=x \frac{y^{k+1}}{(k+1) !}\left(\prod_{l=1}^{k+1} f_{n+1-l}\right)(u)$. By Itô's for- 
mula we can write

$$
\begin{aligned}
& F\left(I_{n-k-1}^{S}(t), W(t), t\right)=\int_{0}^{t} \frac{(W(s))^{k+1}}{(k+1) !}\left(\prod_{l=1}^{k+1} f_{n+1-l}\right)(s) d I_{n-k-1}^{S}(s) \\
& \quad+\int_{0}^{t} I_{n-k-1}^{S}(s) \frac{(W(s))^{k}}{k !}\left(\prod_{l=1}^{k+1} f_{n+1-l}\right)(s) d W(s) \\
& \quad+\int_{0}^{t} I_{n-k-1}^{S}(s) \frac{(W(s))^{k+1}}{(k+1) !}\left(\prod_{l=1}^{k+1} f_{n+1-l}\right)^{\prime}(s) d s \\
& +\frac{1}{2} \int_{0}^{t} \frac{(W(s))^{k}}{k !}\left(\prod_{l=1}^{k+1} f_{n+1-l}\right)(s) d\left[I_{n-k-1}^{S}(s), W(s)\right] .
\end{aligned}
$$

Putting together (11) and (12) and using that from Proposition 3.2

$$
\begin{aligned}
& \frac{1}{2} \int_{0}^{t} \frac{(W(s))^{k}}{k !}\left(\prod_{l=1}^{k+1} f_{n+1-l}\right)(s) d\left[I_{n-k-1}^{S}(s), W(s)\right] \\
& =\frac{1}{2} \int_{0}^{t} \frac{W(s)^{k}}{k !}\left(\prod_{l=1}^{k+1} f_{n+1-l}\right)(s) f_{n-k-1}(s) I_{n-k-2}^{S}(s) d s
\end{aligned}
$$

we get that

$$
\begin{aligned}
H_{k}^{S}(t) & =I_{n-k-1}^{S}(t) \frac{(W(t))^{k+1}}{(k+1) !}\left(\prod_{l=1}^{k+1} f_{n+1-l}\right)(t) \\
- & \int_{0}^{t} I_{n-k-1}^{S}(s) \frac{(W(s))^{k+1}}{(k+1) !}\left(\prod_{l=1}^{k+1} f_{n+1-l}\right)^{\prime}(s) d s \\
- & H_{k+1}^{S}(t) .
\end{aligned}
$$

So, from (10) we can write

$$
\begin{aligned}
& I_{n}^{S}(t)=I_{n-1}^{S}(t) W(t) f_{n}(t)-\int_{0}^{t} I_{n-1}^{S}(s) W(s) f_{n}^{\prime}(s) d s-H_{1}^{S}(t) \\
& =I_{n-1}^{S}(t) W(t) f_{n}(t)-\int_{0}^{t} I_{n-1}^{S}(s) W(s) f_{n}^{\prime}(s) d s \\
& \quad-I_{n-2}^{S}(t) \frac{(W(t))^{2}}{2 !} f_{n}(t) f_{n-1}(t)+\int_{0}^{t} I_{n-2}^{S}(s) \frac{(W(s))^{2}}{2 !}\left(f_{n} f_{n-1}\right)^{\prime}(s) d s \\
& \quad+H_{2}^{S}(t)
\end{aligned}
$$

and the proof finishes iterating (13) $n-1$ times. 


\section{Proof of the main result}

We begin with a technical lemma where we obtain an expression for $J_{n}^{(m)}=$ $J_{n}^{(m)}\left(f_{1}, \ldots, f_{n}\right)(t)$, similar to the expression given in Proposition 3.3 for the multiple Stratonovich integral. This result is also inspired by the paper [13].

Lemma 4.1. Assume that for each $i \in\{1, \ldots, n\}, f_{i}$ has continuous derivatives in $[0, T]$. Then

$$
\begin{aligned}
& J_{n}^{(m)}(t)=\sum_{k=1}^{n}(-1)^{k+1} J_{n-k}^{(m)}(t) \frac{\left(W^{(m)}(t)\right)^{k}}{k !}\left(\prod_{l=1}^{k} f_{n+1-l}\right)(t) \\
& +\sum_{k=1}^{n}(-1)^{k} \int_{0}^{t} J_{n-k}^{(m)}(s) \frac{\left(W^{(m)}(s)\right)^{k}}{k !}\left(\prod_{l=1}^{k} f_{n+1-l}\right)^{\prime}(s) d s,
\end{aligned}
$$

for any $t \in[0, T]$ and with $J_{0}^{(m)}(t)=1$.

Proof: Consider the function $F(x, y, u)=x y f_{n}(u)$. Since the functions are of bounded variation

$$
\begin{aligned}
& F\left(J_{n-1}^{(m)}(t), W^{(m)}(t), f_{n}(t)\right)=\int_{0}^{t} W^{(m)}(s) f_{n}(s) d J_{n-1}^{(m)}(s) \\
& \quad+\int_{0}^{t} J_{n-1}^{(m)}(s) f_{n}(s) d W^{(m)}(s)+\int_{0}^{t} J_{n-1}^{(m)}(s) W^{(m)}(s) f_{n}^{\prime}(s) d s
\end{aligned}
$$

From (3), (14) can be written

$$
\begin{aligned}
& J_{n}^{(m)}(t)=J_{n-1}^{(m)}(t) W^{(m)}(t) f_{n}(t) \\
& -\int_{0}^{t} W^{(m)}(s) f_{n}(s) d J_{n-1}^{(m)}(s)-\int_{0}^{t} J_{n-1}^{(m)}(s) W^{(m)}(s) f_{n}^{\prime}(s) d s,
\end{aligned}
$$

Thus, now we need to study

$$
\int_{0}^{t} W^{(m)}(s) f_{n}(s) d J_{n-1}^{(m)}(s)
$$

Actually, we will study the more general integral

$$
H_{k}(t):=\int_{0}^{t} \frac{\left(W^{(m)}(s)\right)^{k}}{k !}\left(\prod_{l=1}^{k} f_{n+1-l}\right)(s) d J_{n-k}^{(m)}(s) .
$$


Consider the function $F(x, y, u)=x \frac{y^{k+1}}{(k+1) !}\left(\prod_{l=1}^{k+1} f_{n+1-l}\right)(u)$. Since the functions are of bounded variation we can write

$$
\begin{aligned}
& F\left(J_{n-k-1}^{(m)}(t), W^{(m)}(t), t\right)=\int_{0}^{t} \frac{\left(W^{(m)}(s)\right)^{k+1}}{(k+1) !}\left(\prod_{l=1}^{k+1} f_{n+1-l}\right)(s) d J_{n-k-1}^{(m)}(s) \\
& \quad+\int_{0}^{t} J_{n-(k+1)}^{(m)}(s) \frac{\left(W^{(m)}(s)\right)^{k}}{k !}\left(\prod_{l=1}^{k+1} f_{n+1-l}\right)(s) d W^{(m)}(s) \\
& \quad+\int_{0}^{t} J_{n-(k+1)}^{(m)}(s) \frac{\left(W^{(m)}(s)\right)^{k+1}}{(k+1) !}\left(\prod_{l=1}^{k+1} f_{n+1-l}\right)^{\prime}(s) d s
\end{aligned}
$$

Then, using the definition of $F$ and (16) we have that

$$
\begin{aligned}
H_{k}(t)= & \int_{0}^{t} \frac{\left(W^{(m)}(s)\right)^{k}}{k !}\left(\prod_{l=1}^{k} f_{n+1-l}\right)(s) d J_{n-k}^{(m)}(s) \\
= & \int_{0}^{t} J_{n-(k+1)}^{(m)}(s) \frac{\left(W^{(m)}(s)\right)^{k}}{k !}\left(\prod_{l=1}^{k+1} f_{n+1-l}\right)(s) d W^{(m)}(s) \\
= & J_{n-(k+1)}^{(m)}(t) \frac{\left(W^{(m)}(t)\right)^{k+1}}{(k+1) !}\left(\prod_{l=1}^{k+1} f_{n+1-l}\right)(t) \\
& \quad-\int_{0}^{t} \frac{\left(W^{(m)}(s)\right)^{k+1}}{(k+1) !}\left(\prod_{l=1}^{k+1} f_{n+1-l}\right)(s) d J_{n-(k+1)}^{(m)}(s) \\
& \quad-\int_{0}^{t} J_{n-(k+1)}^{(m)}(s) \frac{\left(W^{(m)}(s)\right)^{k+1}}{(k+1) !}\left(\prod_{l=1}^{k+1} f_{n+1-l}\right)^{\prime}(s) d s \\
= & J_{n-(k+1)}^{(m)}(t) \frac{\left(W^{(m)}(t)\right)^{k+1}}{(k+1) !}\left(\prod_{l=1}^{k+1} f_{n+1-l}\right)(t) \\
\quad-H_{k+1}(t) & \quad-\int_{0}^{t} J_{n-(k+1)}^{(m)}(s) \frac{\left(W^{(m)}(s)\right)^{k+1}}{(k+1) !}\left(\prod_{l=1}^{k+1} f_{n+1-l}\right)^{\prime}(s) d s .
\end{aligned}
$$

Iterating the same argument $n-1$ we finish the proof.

We can give now the proof of the main theorem.

Proof of Theorem 2.1; We have to check that, almost surely

$$
\lim _{m \rightarrow \infty} J_{n}^{(m)}\left(f_{1}, \ldots, f_{n}\right)(t)=I_{n}^{S}\left(f_{1}, \ldots, f_{n}\right)(t),
$$

for any $t \in[0, T]$. We use an induction argument. Let us introduce our hypotesis of induction: 
$\left(\bar{H}_{j}\right)$ For any $l \leq j$, almost surely

$$
\lim _{m \rightarrow \infty} J_{j}^{(m)}\left(f_{1}, \ldots, f_{j}\right)(t)=I_{j}^{S}\left(f_{1}, \ldots, f_{j}\right)(t),
$$

for all $t \in[0, T]$. Moreover, for almost all $\omega$ there exists $m_{j}(\omega)$ and $K_{j}(\omega)$, both finite, such that for all $m>m_{j}$ and all $s \in[0, T]$ and all $l \leq j, J_{l}^{(m)}(s, \omega) \leq K_{j}(\omega)$.

Let us study $\left(\bar{H}_{1}\right)$ first. It is a well-known that almost surely

$$
\lim _{m \rightarrow \infty} J_{1}^{(m)}(t)=\lim _{m \rightarrow \infty} \int_{0}^{t} W^{(m)}(s) d W^{(m)}(s)=\int_{0}^{t} W(s) d^{\circ} W(s)=I_{1}^{(S)}(t),
$$

for all $t \in[0, T]$. On the other hand, from Lemma 4.1 we have that

$$
J_{1}^{(m)}(t)=W^{(m)}(t) f_{1}(t)-\int_{0}^{t} W^{(m)}(s) f_{1}^{\prime}(s) d s .
$$

On the other hand, the boundedness of $J_{1}^{(m)}$ is a consequence of the boundedness of $W^{(m)}$ and the continuity of $f_{1}$ and $f_{1}^{\prime}$. So, $\left(\bar{H}_{1}\right)$ is clearly true.

Consider now $j>1$. Let us assume now that $\left(\bar{H}_{j-1}\right)$ is true and we will check that $\left(\bar{H}_{j}\right)$ holds. Using Proposition 3.3 and Lemma 4.1 to get expressions of $I_{S}^{(m)}$ and $J_{j}^{(m)}$, in order to prove the almost sure convergence it is enough to check that for any $k \in\{1, \ldots, j\}$, almost surely

$$
\begin{gathered}
\lim _{m \rightarrow \infty} J_{j-k}^{(m)}(t) \frac{\left(W^{(m)}\right)^{k}}{k !}(t)\left(\prod_{l=1}^{k} f_{j+1-l}\right)(t) \\
=I_{j-k}^{S}(t) \frac{W(t)^{k}}{k !}\left(\prod_{l=1}^{k} f_{j+1-l}\right)(t), \\
\lim _{m \rightarrow \infty} \int_{0}^{t} J_{j-k}^{(m)}(s) \frac{\left(W^{(m)}\right)^{k}}{k !}(s)\left(\prod_{l=1}^{k} f_{j+1-l}\right)^{\prime}(s) d s \\
=\int_{0}^{t} I_{j-k}^{S}(s) \frac{W(s)^{k}}{k !}\left(\prod_{l=1}^{k} f_{j+1-l}\right)^{\prime}(s) d s .
\end{gathered}
$$

for any $t \in[0, T]$. The limit (17) is an evident consequence of $\left(\bar{H}_{j-1}\right)$ and (18) follows easily using again $\left(\bar{H}_{j-1}\right)$ and dominated convergence. Notice that the boundedness of $J_{j}^{(m)}$ is obtained from $\left(\bar{H}_{j-1}\right)$ and Lemma 4.1.

When (H4) is also true, from Proposition 3.3 and Lemma 4.1 we can write 
for $j \in\{1, \ldots, n\}$

$$
\begin{aligned}
& \sup _{s \in[0, T]}\left|J_{j}^{(m)}(s)-I_{j}^{S}(s)\right| \\
& \leq \sum_{k=1}^{n} \sup _{s \in[0, T]}\left|\left(J_{n-k}^{(m)}(s) \frac{W^{(m)}(s)^{k}}{k !}-I_{n-k}^{S}(s) \frac{W(s)^{k}}{k !}\right)\left(\prod_{l=1}^{k} f_{n+1-l}\right)(s)\right| \\
& +\sum_{k=1}^{n} \int_{0}^{T}\left|\left(J_{n-k}^{(m)}(s) \frac{W^{(m)}(s)^{k}}{k !}-I_{n-k}^{S}(s) \frac{W(s)^{k}}{k !}\right)\left(\prod_{l=1}^{k} f_{n+1-l}\right)^{\prime}(s)\right| d s \\
& \leq \sum_{k=1}^{n} \sup _{s \in[0, T]}\left|J_{n-k}^{(m)}(s)\right| \sup _{s \in[0, T]}\left|\frac{W^{(m)}(s)^{k}}{k !}-\frac{W(s)^{k}}{k !}\right| \sup _{s \in[0, T]}\left|\left(\prod_{l=1}^{k} f_{n+1-l}\right)(s)\right| \\
& +\sum_{k=1}^{n} \sup _{s \in[0, T]} \mid\left(J_{n-k}^{(m)}(s)-I_{n-k}^{S}(s)\left|\sup _{s \in[0, T]}\right| \frac{W(s)^{k}}{k !}\left|\sup _{s \in[0, T]}\right|\left(\prod_{l=1}^{k} f_{n+1-l}\right)(s) \mid\right. \\
& +\sum_{k=1}^{n} \int_{0}^{T}\left|J_{n-k}^{(m)}(s)\right| \times\left|\frac{W^{(m)}(s)^{k}}{k !}-\frac{W(s)^{k}}{k !}\right| \times\left|\left(\prod_{l=1}^{k} f_{n+1-l}\right)^{\prime}(s)\right| d s \\
& +\sum_{k=1}^{n} \int_{0}^{T} \mid\left(J_{n-k}^{(m)}(s)-I_{n-k}^{S}(s)|\times| \frac{W(s)^{k}}{k !}|\times|\left(\prod_{l=1}^{k} f_{n+1-l}\right)^{\prime}(s) \mid d s .\right.
\end{aligned}
$$

Using the equality $\left(x^{k}-y^{k}\right)=(x-y) \sum_{k=1}^{n} x^{n-k} y^{k-1}$ we have that

$$
\left|W^{(m)}(s)^{k}-W(s)^{k}\right| \leq\left|W^{(m)}(s)-W(s)\right| \sum_{k=1}^{n}\left|W^{(m)}(s)\right|^{n-k}|W(s)|^{k-1} .
$$

Then using that

1. $\sup _{s \in[0 . T]}|W(s)|<+\infty$ almost surely,

2. $\sup _{s \in[0, T]}\left(\left|\left(\prod_{l=1}^{k} f_{n+1-l}\right)(s)\right|+\left|\left(\prod_{l=1}^{k} f_{n+1-l}\right)^{\prime}(s)\right|\right)<\infty$

3. for almost all $\omega$ there exists $m_{n}(\omega)$ and $K_{n}(\omega)$, both finite, such that for all $m>m_{n}$ and all $s \in[0, T]$ and all $l \leq n, J_{l}^{(m)}(s, \omega) \leq K_{j}(\omega)$.

4. $\lim _{m \rightarrow \infty} \sup _{s \in[0, T]}\left|W^{(m)}(s)-W(s)\right|=0$ almost surely and doing an interation procedure we can finish easily the proof of the theorem.

Proof of Theorem 2.2 The proof follows easily from Theorem 2.1 and the strong convergence result in [11. 


\section{References}

[1] Bardina, X.; Binotto, G. and Rovira, C. The complex Brownian motion as a strong limit of processes constructed from a Poisson process.J. Math. Anal. Appl. Vol.444 (2016), no. 1, 700-720.

[2] Bardina, X.; Ferrante, M. and Rovira, C. Collect. Math. (2019). https://doi.org/10.1007/s13348-019-00263-4

[3] Bardina, X.; Jolis, M. Weak convergence to the multiple Stratonovich integral Stochastic Processes and their Applications Vol.90 (2000), 277-300.

[4] Csörgo, M.; Horváth, L. Rate of convergence of transport processes with an application to stochastic differential equations. Probab. Theory Related Fields Vol.78 (1988), no. 3, 379-387.

[5] Garzón, J.; Torres, S.; Tudor, C.A. A strong convergence to the Rosenblatt process Journal of Mathematical Analysis and Applications 391 (2012), 630-647.

[6] Garzón, J.; Gorostiza, L. G.; León, J. A. A strong uniform approximation of fractional Brownian motion by means of transport processes. Stochastic Process. Appl. Vol.119 (2009), no. 10, 3435-3452.

[7] Garzón, J.; Gorostiza, L. G.; León, J. A strong approximation of subfractional Brownian motion by means of transport processes. In: Malliavin calculus and stochastic analysis, 335-360, Springer Proc. Math. Stat., 34, Springer, New York, (2013).

[8] Garzón, J.; Gorostiza, L. G.; León, J. Approximations of Fractional Stochastic Differential Equations by means of transport processes. Commun. Stoch. Anal. Vol.5. No.3 (2011), 433-456.

[9] Gorostiza, L.G. and Griego, R.J. Strong approximation of diffusion processes by transport processes. Journal of Mathematics of Kyoto University Vol.19 (1979), No. 1, 91-103.

[10] Gorostiza, L.G. and Griego, R.J. Rate of convergence of uniform transport processes to Brownian motion and application to stochastic integrals. Stochastics Vol. 3 (1980), 291-303.

[11] Griego, R.J., Heath, D. and Ruiz-Moncayo, A. Almost sure convergence of uniform trasport processes to Brownian motion. Ann. Math. Stat. Vol. 42 (1971), No. 3, 1129-1131.

[12] Kloeden, P.E. and Platen, E. Relations beteween multiple Itô and Stratonovich integrals. Stochastic Analysis and Applications . Vol 3 (1991), 311-321.

[13] Wong, E. and Zakai, M. On the convergence of ordinary integrals to stochastic integrals. Ann. Math. Statist. Vol 36, no. 5 (1965), 1560-1564. 